\title{
GENIE: high-resolution study of debris disks
}

\author{
Olivier Absil ${ }^{1}$, Jean-Charles Augereau ${ }^{2,3}$, Roland den Hartog ${ }^{4}$, Emilie \\ Herwats $^{1,2}$, Philippe Gondoin ${ }^{4}$, and Malcolm Fridlund ${ }^{4}$ \\ 1 Institut d'Astrophysique et de Géophysique, Université de Liège, Belgium \\ ${ }^{2}$ Laboratoire d'Astrophysique de Grenoble, France \\ 3 Sterrewacht Leiden, University of Leiden, The Netherlands \\ 4 ESA/ESTEC, Noordwijk, The Netherlands
}

\section{Simulated observations of the dust disk around $\zeta$ Lep}

In order to evaluate the scientific potential of GENIE [1] in the context of debris disks, we have simulated future observations of $\zeta$ Lep (A2V, $21.5 \mathrm{pc}$ ), a prototypical Vega-type star expected to harbour warm dust in the inner part of its debris disk [2].

We have used the 3D debris disk model developed by Augereau et al. [3] to compute synthetic L'-band images of the optically thin dust disk around $\zeta$ Lep, using a variety of physical parameters. Both the thermal emission and the scattered light are included in the model. Two types of grains have been considered: smoothed astronomical silicates (Weingartner \& Draine 2000) and cold-coagulation type grains ( $\mathrm{Li} \&$ Lunine 2003) with a porosity up to $90 \%$ when the $\mathrm{H}_{2} \mathrm{O}$-dominated ices are sublimated ( $\left.<6 \mathrm{AU}\right)$. The grain size distribution is assumed to follow an $a^{-3.5}$ power law between a minimum and maximum grain size, and the surface density to fade with the distance from the star as $r^{-0.6}$. Sublimation happens at $1700 \mathrm{~K}$, at a distance that depends on the grain composition and size. For each particular grain distribution, we compute the SED of the dust disk and adjust the surface density so that our SED gives the best possible fit to the observed mid-IR excesses. Synthetic images of the disk are then generated for different inclination angles.

The simulated observations are obtained with the GENIEsim software [4], using various AT-AT baselines ranging between 8 and $80 \mathrm{~m}$ on the main EastWest VLTI track. Our simulations take into account all the expected noise sources, including residual atmospheric turbulence after the control loops. Each individual observation consists in a $1000 \mathrm{~s}$ exposure, followed by the observation of a calibrator star with similar H-K-L magnitudes ( $\eta$ Lep, F1V at $15 \mathrm{pc}$ ) to evaluate and subtract the contribution of instrumental leakage. The contribution of geometric leakage, due to the finite extent of the stellar disk, is removed analytically, using a surface brightness model developed by Kervella et al. (A\&A 426, 2004) to evaluate the stellar diameter (estimated LD diameter: $0.756 \pm 0.015$ mas). The final output of the nuller is the nulling ratio, measured as the ratio between the constructive and destructive outputs. 


\section{Influence of the disk parameters on the nulling ratio}

The evolution of the sublimation radius as a function of grain size is given in Fig. 1. Large grains are less susceptible to sublimation and are thus more concentrated close to the star. The global SED of the disk is therefore shifted towards shorter wavelengths, inducing a larger flux in the L' band for the same mid-IR excess. This leads to a larger amount of transmitted light in the nulled output. Moreover, the nulling ratio reaches a local maximum when the chosen baseline makes the first bright fringe coincide with the bright inner rim of the disk $\left(\lambda / 2 B=r_{\text {in }}\right)$, which happens at $80 \mathrm{~m}$ for the largest grains and at $24 \mathrm{~m}$ for the smallest grains.

The influence of grain type is investigated in Fig. 2, using either silicate or porous grains. The latter are less effective than silicate grains to re-emit a thermal radiation. A larger density of porous grains is thus required to produce the same mid-IR excess, which leads to an increased L'-band flux with respect to the silicate grains. The nulling curve is thus translated upwards. A second effect comes from the better resistance of silicate grains to sublimation due to their compact nature. The thermal emission is therefore more concentrated close to the star in the case of silicate grains. This can be clearly seen in Fig. 2: the first maximum in the nulling ratio is obtained for a smaller baseline in the case of porous grains. This means that the first bright fringe reaches more rapidly the inner rim of the disk as the baseline is increased, revealing a larger inner rim in the case of porous grains.

Finally, another important effect on the nulling ratio is the orientation under which the dust disk is seen. We have simulated observations of inclined disks, using angles between $10^{\circ}$ (highly inclined) and $90^{\circ}$ (face-on). The baselines are perpendicular to the major axis of the projected image of the disk (fringe pattern parallel with the major axis). Highly inclined disks are less transmitted by the nuller because most of the disk emission falls onto the dark fringe, especially for short baselines. This effect is clearly evidenced in Fig. 2, showing a great sensitivity of the nuller to inclination. Note that the contribution of scattered light (only a few percent of the total disk emission in L' band) is more extended and thus less affected by inclination.

In conclusion, an L'-band nuller such as GENIE is well adapted to study the thermal emission from the inner parts of debris disks. A few baselines are sufficient to constrain the grain composition and major disk parameters.

\section{References}

1. P. Gondoin, O. Absil, R. den Hartog et al: Proc. SPIE 5491, 775 (2004)

2. S. Fajardo-Acosta, C. Telesco and R. Knacke: AJ 115, 2101 (1998)

3. J.-C. Augereau, A.-M. Lagrange, D. Mouillet et al.: A\&A 348, 557 (1999)

4. O. Absil, R. den Hartog, C. Erd et al: GENIEsim, the GENIE simulation software. In: Towards Other Earths, ed B. Battrick (ESA-SP 539, 2003), 317 

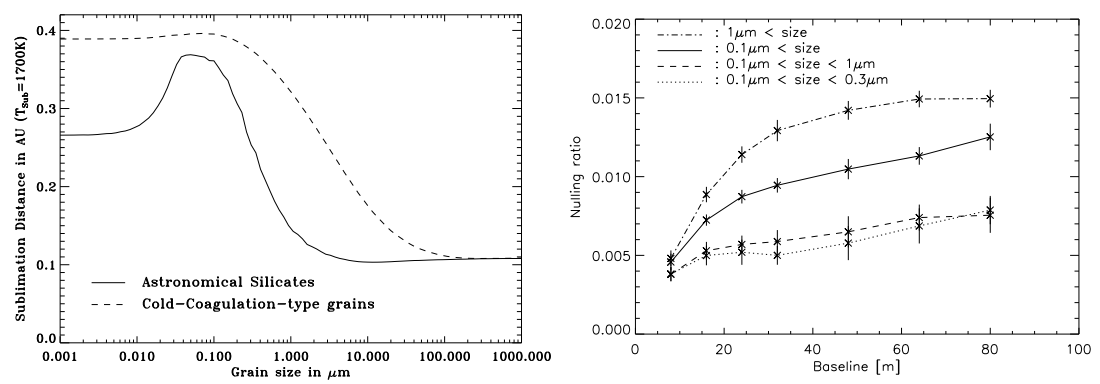

Fig. 1. Left: Sublimation distance of dust grains as a function of their size, for two types of grains around $\zeta$ Lep. The heating process is more efficient for small grains, which reach the sublimation temperature further from the star. Right: Influence of the size distribution of dust grains on the observed nulling ratio with GENIE, using different AT-AT baselines. Error bars take into account all noise sources including calibration processes. The disk is supposed to be face-on.
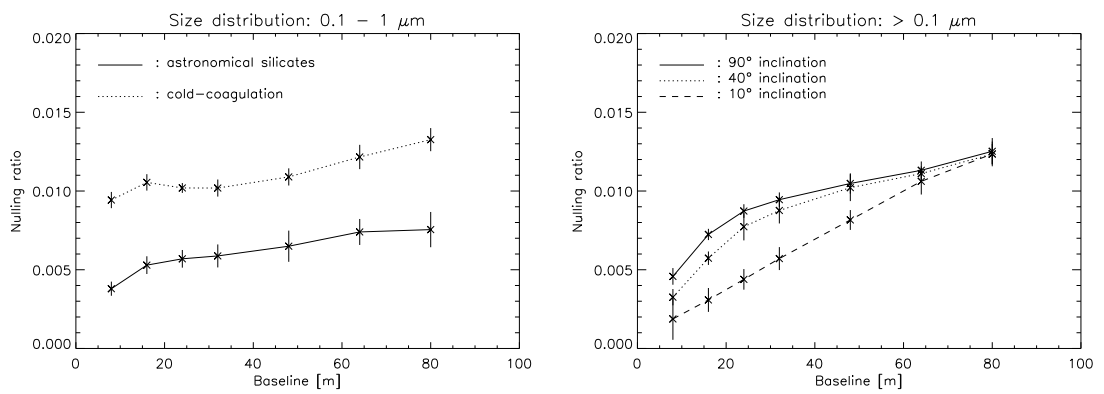

Fig. 2. Left: Influence of the physical composition of the dust grains on the observed nulling ratio. Cold-coagulation grains are highly porous and thus more efficiently heated. The inner rim is therefore shifted outwards in this case. Right: Influence of the disk inclination on the nulling ratio. We have assumed the major axis of the projected dust disk to be aligned with the main AT-AT track in this simulation. Highly inclined disks are more attenuated by the central dark fringe. 